\title{
QRS Duration as a Marker of Microvascular Reperfusion Assessed by Myocardial Blush Grade in Acute ST Elevation Myocardial Infarction Patients Undergoing Primary Percutaneous Intervention
}

\begin{abstract}
a Department of cardiology, Benha faculty of medicine, Benha University, Egypt.

b Department of cardiology, National heart institute, Egypt
\end{abstract}

Wael Tawfik ${ }^{\mathrm{a}}$, Ahmed Ramzy $^{\mathrm{a}}$, Mohamed Saed $^{\mathrm{a}}$, Yasser Elsayed ${ }^{\mathrm{b}}$

Correspondence to: Wael Tawfik, Department of cardiology, Benha faculty of medicine, Benha University, Egypt.

Email:

wtwfik@hotmail.com

Received: 17 November 2020

Accepted: 20 December 2020

\begin{abstract}
:
Background: Primary percutaneous coronary intervention (PCI) is the treatment of choice for patients presenting with ST-elevation myocardial infarction (STEMI). In patients with STEMI, QRS prolongation, in the absence of bundle branch blocks, was considered mainly a dynamic phenomenon likely to be improved by successful reperfusion. Aim: To evaluate the role of QRS duration change following primary angioplasty in patients presented with STEMI as a marker of microvascular reperfusion assessed by myocardial blush grade (MBG). Methods: Fifty patients with STEMI were included with this study, 37 males and 13 females, their age ranged between 32 to 74 years. ECG was done to all patients at presentation and after primary PCI to asses QRS duration change .Angiographic assessment of myocardium supplied by infarct related artery was performed using MBG. According to MBG patients were classified into two groups: group (1), with good myocardial reperfusion MBG (2-3) and group (2), with poor myocardial reperfusion MBG (0-1). Results: Although there is no difference between two groups according to sex, smoking, hypertension and diabetes,
\end{abstract} patients with higher age were at risk of poor tissue reperfusion $(\mathrm{p}=0.049)$. Patients with poor tissue reperfusion had longer QRS duration on presentation and after PCI compared to normal perfusion group $(P<0.001)$.There was significant positive correlation between $\mathrm{QRS}$ narrowing and left ventricular ejection fraction, on the other hand there was significant negative correlation between QRS narrowing and door to balloon time. Conclusion: QRS duration change post angioplasty is a strong marker of myocardial reperfusion in patients presenting with STEMI.

Key words: primary angioplasty, coronary flow, STEMI 



\section{Introduction}

Primary percutaneous coronary intervention (PCI) is the treatment of choice for patients presenting with ST-elevation myocardial infarction (STEMI). Patency of the infarctrelated artery (IRA) along with a good microvascular flow is the ultimate goal of reperfusion therapy (1). Nevertheless, the patency of the IRA does not guarantee the salvage of the myocardium under risk of ischemia (2).

The prolongation of the QRS duration in ST elevation myocardial infarction (STEMI) has been identified as an independent predictor of adverse outcome (3).

QRS narrowing following PCI indicates adequate microvascular reperfusion and persistence of QRS prolongation is associated with poor microvascular flow (1).

While ST-segment resolution is a predictor myocardial perfusion and cell membrane integrity, myocardial blush grade (MBG) relates to myocardial perfusion and microvascular patency. MBG has been found to be a predictor [independent of thrombolysis in myocardial infarction (TIMI) flow] of both short and long-term mortality in patients with acute myocardial infarction who underwent primary angioplasty (4).

In this study, we evaluated the role of QRS duration change following primary angioplasty in patients presented with STEMI as a marker of microvascular reperfusion assessed by MBG.

\section{Patients and methods}

This is a prospective observational study, conducted at NHI" National Heart Institute". The protocol of the study was approved by the local ethical committee.

The study included fifty patients presented by STEMI within 12 hours of symptom onset to NHI" National Heart Institute" from November 2017 to June 2018, and treated by primary PCI. STEMI was defined as chest pain typical for myocardial infarction of at least 30 minutes duration and $<6$ hours, and new ST-segment elevation in two contiguous leads with the cutoff points $\geq 0.2 \mathrm{mV}$ in men or $\geq 0.15 \mathrm{mV}$ in women in leads V2-V3 and/or $\geq 0.1 \mathrm{mV}$ in other leads on the 12-lead ECG. Patients with bundle branch block, post arrest, electrolyte disturbance, $\mathrm{LVH}$ and cardiogenic shock were excluded.

After detailed clinical examination, all patients received $300 \mathrm{mg}$ chewable acetyl salicylate, $600 \mathrm{mg}$ clopidogrel (or 180mg ticagrelol), IV morphine and nitrates, blood samples drawn from all patients to asses serum creatinine, complete blood count and cardiac enzymes. 


\section{Electrocardiogram}

A 12-lead ECG with a paper speed of $50 \mathrm{~mm} / \mathrm{s}$ and amplification of $10 \mathrm{~mm} / \mathrm{mV}$ was recorded at admission as well as at the immediate postprocedure and $60 \mathrm{~min}$ following primary PCI. The duration of QRS was calculated manually by two investigators, and was measured from the beginning of the earliest to the end of the last QRS deflection, and all measurements were obtained from the infarct-related artery (IRA) lead with highest ST segment elevation. The average QRS duration of two observers was utilized for analysis and Change in QRS duration was calculated by subtracting postangioplasty QRS duration from preangioplasty QRS duration.

\section{Primary PCI}

All patients underwent primary percutaneous coronary intervention within 90 mins of diagnosis establishment. At the start of the procedure, 100U/kg of heparin was given, all patients underwent initial balloon angioplasty and coronary stenting, when felt to be needed by the primary operator.

The final coronary angiogram run was long enough to visualize the venous phase of the contrast passage, and MBG was then calculated manually by two blinded experienced interventional cardiologists based on the assessment of contrast opacification of the myocardial territory subtended by the IRA. A third reader's opinion was used in case of any discordance. $\mathrm{MBG}$ was graded as $0=$ no myocardial blush or contrast density, $1=$ minimal myocardial blush or contrast density, $2=$ myocardial blush or contrast density which exists to a lesser extent and its clearance is diminished compared with noninfarct-related coronary artery, and $3=$ normal myocardial blush or contrast density comparable with that obtained during angiography of a contralateral or ipsilateral non-infarct-related coronary artery (5).

Echocardiography was done to all patients within 24 hour of the index procedure; left ventricular ejection fraction (LVEF) was assessed using modified Simpson's method. The relation between change in QRS duration and MBG was analyzed. The study protocol was approved by local Ethics Committee and informed consent was obtained from all patients.

\section{Statistical analysis:}

SPSS 18.0 (SPSS Inc., Chicago, Illinois, USA) was used for statistical analyses. The normality of the distribution of continuous variables was evaluated using the Kolmogorov-Smirnov test. For continuous variables, the Student t-test was used to test differences between groups. For correlation 
analysis, Spearman's correlation analysis was considered statistically significant. Continuous variables were expressed as mean \pm SD and categorical variables were expressed as percentages.

\section{Results}

Fifty patients included in the study were divided into two groups according to the reperfusion status, as assessed by the MBG; group 1 with $\mathrm{MBG}$ of 2-3 ( $\mathrm{n}=37)$, considered as the reperfusion group and group 2 with MBG of 0-1 (n =13), which constituted impaired reperfusion.

The baseline clinical, echocardiographic, and angiographic parameters are shown in Table 1. The two groups were similar in terms of gender, hypertension, and diabetes status, ( $\mathrm{p}>$ 0.05).Whereas there was statistically significant difference between two groups as regard age being older age was present in group 2 patients with impaired perfusion ( $\mathrm{P}$ $=0.049)$. The pain to balloon time and door to balloon time were significantly higher in group 2 with impaired perfusion in comparison with group 1 with normal perfusion. In terms of echocardiographic parameters, LVEF was significant lower in group 2 with impaired perfusion .As regard IRA there was no significant difference between 2 groups.

QRS duration study. Table (2) used. Two-tailed $\mathrm{P}$ values less than 0.05 were At presentation QRS duration was longer in group 2 (impaired perfusion group) compared to group 1 (normal perfusion group) (93.85 \pm $12.61 \mathrm{~m} \mathrm{sec}$ vs $86.49 \pm 10.60 \mathrm{msec}, \mathrm{P}=0.024$, respectively ) .

QRS duration was found to be significantly longer in group 2 compared to group 1 at both the immediate ECG $(98.08 \pm 11.64$ vs $69.59 \pm$ $10.70 \mathrm{msec}, \mathrm{p}<0.001$, respectively) and at the 60th minute ECG $(105.0 \pm 10.21$ vs $67.16 \pm$ 9.76 msec, $\mathrm{p}<0.001$, respectively) post angioplasty.

\section{Mean QRS duration change "narrowing"}

There was highly statistically significance difference between 2 groups regarding mean change in ORS duration immediately and 60 min after PCI as shown in Table (3).

Correlation between QRS narrowing at the post-angioplasty 60th minute ECG and LVEF and TIMI flow.

After analysis of the total sample $(n=50)$, we observed significant positive correlation between QRS duration change on admission and 1 hour post angioplasty and LVEF ( $\mathrm{r}$ $=0.718 ; \mathrm{p}<0.001)$ as shown in figure $(\mathbf{1})$.

Also we observed a significant and positive correlation between QRS duration Change on admission and 1 hour post angioplasty and TIMI flow ( $\mathrm{r}=0.706 ; \mathrm{p}<0.001)$ as shown in figure (2), There was a negative correlation between the door -to-balloon time and the 
60th minute $(r=\ldots 0.273 ; \mathrm{p}=0.055)$ QRS narrowing .figure(3)

Table 1: The baseline clinical, echocardiographic, and angiographic parameters.

\begin{tabular}{llll}
\hline & $\begin{array}{l}\text { Group 1 (MBG 2-3) } \\
{[\mathbf{n = 3 7}]}\end{array}$ & $\begin{array}{l}\text { Group 2 (MBG 0-1) } \\
{[\mathbf{n}=\mathbf{1 3}]}\end{array}$ & P \\
\hline Age (years) [mean \pm SD] & $51.24 \pm 9.95$ & $57.69 \pm 9.89$ & 0.049 \\
Male gender [n (\%)] & $28(75.7)$ & $9(6.2)$ & 0.719 \\
Diabetes [n (\%)] & $15(40.5)$ & $8(61.5)$ & 0.191 \\
Hypertension [n (\%)] & $14(37.8)$ & $8(61.5)$ & 0.139 \\
Smoking [n (\%)] & $26(70.3)$ & $9(69.2)$ & 1.000 \\
Pain to balloon time.(hours) [mean \pm SD] & $4.41 \pm 1.38$ & $10.23 \pm 1.79$ & $<0.001$ \\
Door to balloon time (mins) [mean \pm SD] & $35.70 \pm 15.74$ & $61.23 \pm 18.18$ & $<0.001$ \\
LVEF (\%) [mean \pm SD] & $52.51 \pm 6.40$ & $44.77 \pm 2.59$ & $<0.001$ \\
IRA [n (\%)] & & & \\
LAD & $23(62.2)$ & $5(38.5)$ & 0.309 \\
LCX & $3(8.1)$ & $2(15.4)$ & \\
RCA & $13(29.7)$ & $6(46.2)$ & \\
TIMI flow at the end of PCI [n (\%)] & & $69.2 \%$ & $<0.001$ \\
TIMI I & $0 \%$ & $30.8 \%$ & \\
TIMI II & $21.6 \%$ & $0 \%$ & \\
TIMI III & 78.4 & \\
\hline
\end{tabular}

LVEF, left ventricle ejection fraction; IRA, infarct-related artery; LAD, left anterior descending; LCX, left circumflex; PCI, percutaneous coronary intervention; RCA, right coronary artery; TIMI, thrombolysis in myocardial infarction; MBG, myocardial blush grade.

Table (2): Comparison between the two studied groups according to QRS duration

\begin{tabular}{llll}
\hline & $\begin{array}{l}\text { Group 1 } \\
\text { MBG (2-3) }\end{array}$ & $\begin{array}{l}\text { Group 2 } \\
\text { MBG (0-1) }\end{array}$ & P \\
\hline $\begin{array}{l}\text { QRS duration on admission (msec) [mean } \pm \\
\text { SD] }\end{array}$ & $86.49 \pm 10.60$ & $93.85 \pm 12.61$ & 0.024 \\
$\begin{array}{l}\text { QRS duration immediately after PCI } \\
\text { (msec) [mean } \pm \text { SD] }\end{array}$ & $69.59 \pm 10.70$ & $98.08 \pm 11.64$ & $<0.001$ \\
$\begin{array}{l}\text { QRS duration 60 min after PCI (msec) } \\
\text { [mean } \pm \text { SD] }\end{array}$ & $67.16 \pm 9.76$ & $105.0 \pm 10.21$ & $<0.001$ \\
\hline
\end{tabular}

Table (3): Comparison between the two studied groups according to QRS duration (change)

\begin{tabular}{llll}
\hline QRS duration change & $\begin{array}{l}\text { Group 1 } \\
\text { MBG (2-3) }\end{array}$ & $\begin{array}{l}\text { Group 2 } \\
\text { MBG (0-1) }\end{array}$ & P \\
\hline $\begin{array}{l}\text { QRS narrowing immediately after PCI } \\
\text { (msec) [mean } \pm \text { SD] }\end{array}$ & $16.89 \pm 9.38$ & $-4.23 \pm 4.49$ & $<0.001$ \\
$\begin{array}{l}\text { QRS narrowing at the post-angioplasty } \\
\text { 60th minute ECG(msec) [mean } \pm \text { SD] }\end{array}$ & $19.32 \pm 10.15$ & $-11.15 \pm 5.46$ & $<0.001$ \\
\hline
\end{tabular}


Benha medical journal vol.38, issue 2, 2021

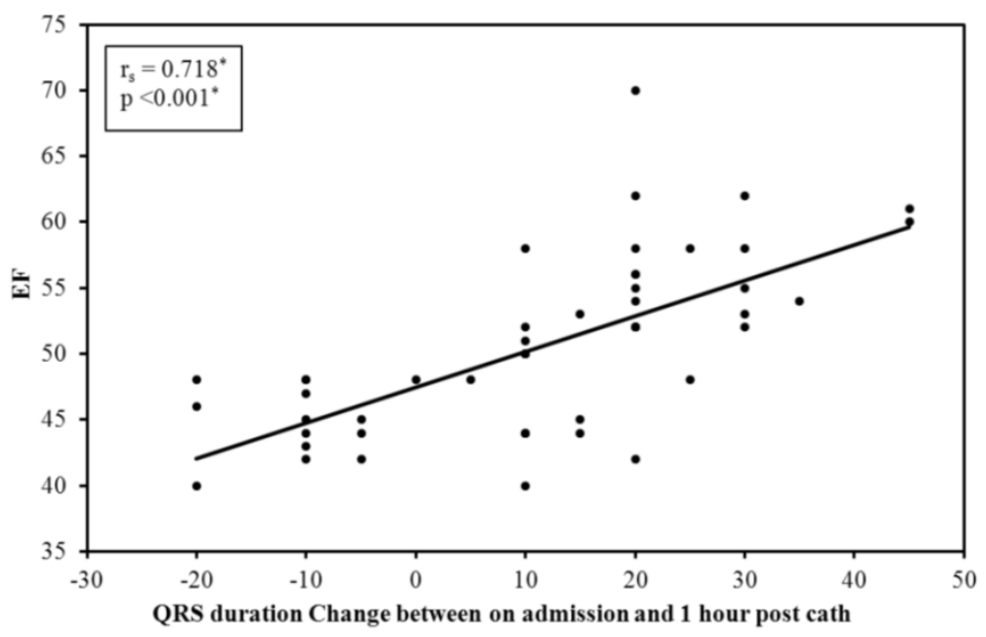

Figure (1): Correlation between QRS duration Change between on admission and 1 hour post cath and EF in total sample $(n=50)$

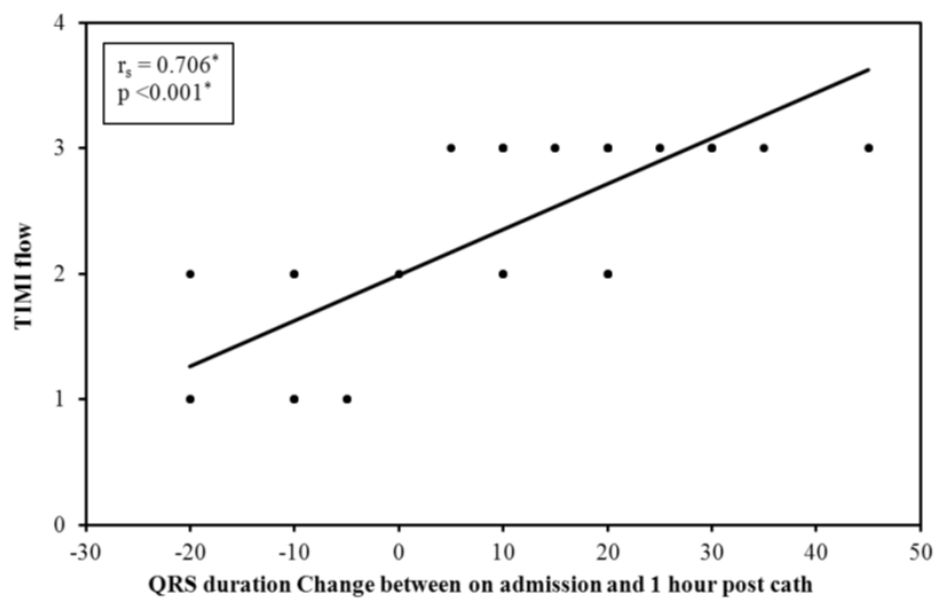

Figure (2): Correlation between QRS duration Change between on admission and 1 hour post cath and TIMI flow in total sample $(n=50)$

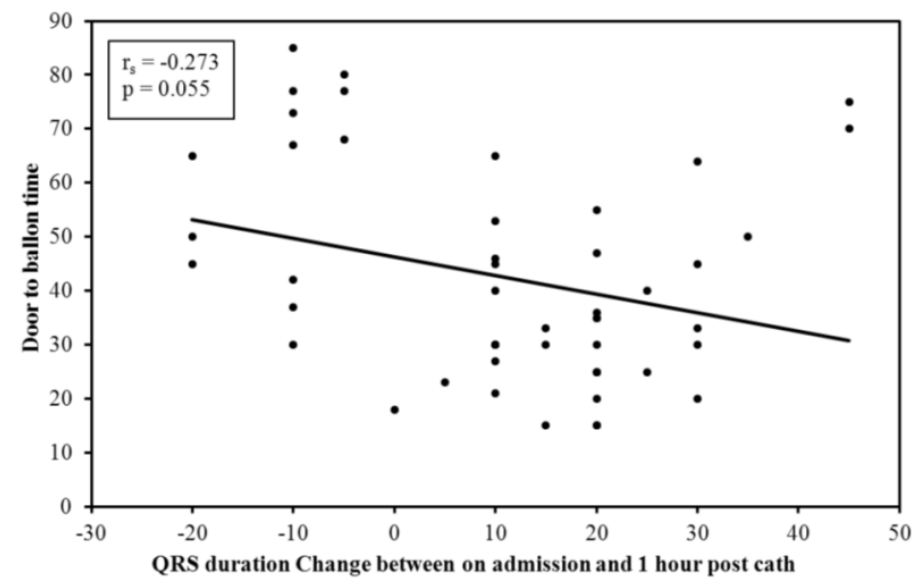

Figure (3): Correlation between QRS duration Change between on admission and 1 hour post cath and Door to balloon time in total sample $(n=50)$ 



\section{Discussion}

In our study we showed the association of QRS duration and myocardial perfusion in patients with acute STEMI following primary angioplasty.

Experimental studies have reported that QRS prolongation is associated with Purkinjeventricular conduction delay caused by myocardial ischemia (5). In the present study the correlation between QRS duration and ischemia duration as assessed by pain to balloon time further supports the idea of ischemia as a mechanism of QRS prolongation.

In the present study, we found statistically significant difference between impaired perfusion group and normal perfusion group as regard LVEF ( $\mathrm{p}<0.001)$. This is in agreement with another study showing the same result as regard EF between two groups of patients (3). In contrast to our results, it was shown that the two groups were statistically similar in terms of admission LVEF and this can be attributed to the time of echocardiography which was performed within 24 hour following primary angioplasty in our study (1).

This study show statistically significance difference between two groups according to pain to balloon time. Other studies showed statistically significant correlation between pain to balloon time and good results regarding $\mathrm{MBG}(2 \& 3)$.

As regard door to balloon time in our study, we found statistically significant difference between the two groups as regard door to balloon time. On the other hand patients with poor tissue reperfusion as assessed by $\mathrm{MBG}$ have longer period of door to balloon time. The same results were observed in LEE et al. study (7).

This study showed the significant relation between narrowing in QRS duration and degree of myocardial reperfusion assessed by MBG in patients with STEMI treated with primary PCI.

In the present study, QRS duration was found to be significantly longer in impaired perfusion compared to normal perfusion group at both the immediate ECG and at the 60th minute ECG post angioplasty ( $\mathrm{p}<0.001)$. Erdogan et al In a study done on 148 patients it was shown that patients in the impaired reperfusion group had a significantly longer QRS duration after primary PCI at both immediate and after $60 \mathrm{~min}$ post angioplasty (2). Similarly, in another study on 100 patients, it was shown that QRS narrowing was significant in patients with a good MBG both at the end of 60th and 90th minutes post angioplasty (8). All these studies further confirm ischemia as a cause of QRS 
prolongation and thus, explain the dynamic nature of QRS changes in these patients.

As regard $\mathrm{QRS}$ duration narrowing and $\mathrm{MBG}$, there was highly statistically significance correlation between QRS duration and MBG, the patients with MBG (2-3) that reveal good tissue reperfusion show significant QRS narrowing immediately and 60 minutes after primary PCI, while patients who had MBG (01) which represent poor tissue reperfusion after procedure doesn't show significant QRS narrowing, no narrowing or even widening of complex immediately and 60 minutes after procedure. A study revealed longer QRS duration after intervention indicating impaired myocardial reperfusion in patients with STEMI (3), and also a similar one showed the same result that QRS duration narrowing is a useful indicator of tissue reperfusion assessed by MBG in patients with STEMI treated by primary PCI (2).

\section{Conclusion:}

QRS duration change post angioplasty is a strong marker of myocardial reperfusion in patients presenting with STEMI.

\section{Abbreviations}

PCI: Percutaneous coronary intervention; STEMI: ST-elevation myocardial infarction; MBG: Myocardial blush grade; IRA: Infarctrelated artery; TIMI: Thrombolysis in myocardial infarction; LVEF: Left ventricular ejection fraction; LAD: Left anterior descending; LCX: Left circumflex; RCA: Right coronary artery.

\section{Reference}

1- Jamal Yusuf , Dipankar Das , Saibal Mukhopadhyay , Sanjay Tyagi. Correlation of QRS duration with myocardial blush grade as a marker of myocardial reperfusion in primary percutaneous coronary intervention. Indian Heart Journal . 2018;70:S359-S364 .

2- Erdogan Ilkay, Fehmi Kacmaz, Orhan Maden , Tolga Aksu ,Mehmet-Timur Selcuk, Ali-Riza Erbay, et al. A new electrocardiographic marker of myocardial reperfusion in patients with acute ST-segment elevation myocardial infarction treated with primary percutaneous intervention: the value of QRS duration. EuroIntervention.2012;7.12:1406-1412.

3- Zülküf Karahan, Bariş Yaylak, Murat Uğurlu, İlyas Kaya, Berzal Uçaman, Önder Öztürk. QRS duration: a novel marker of microvascular reperfusion as assessed by myocardial blush grade in ST elevation myocardial infarction patients undergoing a primary percutaneous intervention. Coron Artery Dis. 2015 Nov; 26(7): 583-586.

4- Hristo T, Morris M. Myocardial Blush Grade: an interventional method for ssessing myocardial perfusion. Isr Med Assoc J. 2008;10:465-467.

5- Van't Hof AWJ, Liem A, Suryapranata H, Hoorntje JCA, Boer MJ, Zijlstra F. Angiographic assessment of myocardial reperfusion in patients treated with primary angioplasty for acute myocardial infarction-myocardial blush grade. Circulation. 1998;97:2302-2306.

6- Mendez C, Mueller WJ, Merideth J, Moe GK. Interaction of trans-membrane potentials in canin Purkinje fibers and at Purkinje fiber-muscle junctions. Circ Res. 1969;24:361-372.

7- Lee CH1, Tai BC, Lau C, Chen Z, Low AF, Teo SG, et al. Relation between Door-to-Balloon Time 
and Microvascular Perfusion as Evaluated by Myocardial Blush Grade, Corrected TIMI Frame Count, and ST-segment Resolution in Treatment of Acute Myocardial Infarction. Journal of interventional cardiology. 2009;22.5: 437-443.
8- Magdy G, Ashmawy H, Mahmoud K, Youssef A. QRS complex duration is a marker of reperfusion in patients presenting with acute ST-segment elevation myocardial infarction. J Cardiovasc Dis Diagn. 2017;5:3.

To cite this article: Wael Tawfik, Ahmed Ramzy, Mohamed Saed, Yasser Elsayed. QRS Duration as a Marker of Microvascular Reperfusion Assessed by Myocardial Blush Grade in Acute ST Elevation Myocardial Infarction Patients Undergoing Primary Percutaneous Intervention. BMFJ 2021; 38(2): 488-496. Pages 1-9. DOI: 10.21608/bmfj.2020.50236.1344 